\title{
1 GHZ GAN RESONANT BODY TRANSISTORS WITH ENHANCED OFF-RESONANCE REJECTION
}

\author{
Laura C. Popa and Dana Weinstein \\ Massachusetts Institute of Technology, Cambridge, Massachusetts, USA
}

\begin{abstract}
We present $1 \mathrm{GHz}$ Gallium Nitride (GaN) Resonant Body Transistors (RBTs) with $>100 \times$ improvement in signal-to-noise ratio relative to previously reported devices. This is realized by strong mechanical coupling of a 1-port resonator with a second resonator in which a High Electron Mobility Transistor is used for sensing. As the first demonstration of GaN RBTs in gold-free technology, this work enables $Q$ s up to 3077 at $1 \mathrm{GHz}$, with $f \cdot Q$ product of $3.1 \times 10^{12}$. We also demonstrate an acoustic transconductance $\left(g_{e m}\right)$ of $72 \mu \mathrm{S},>2 \times$ higher than previously demonstrated GaN RBTs. With such high $Q$ and $g_{e m}$, as well as reduced feedthrough, these GaN RBTs can enable high performance, low phase-noise oscillators monolithically integrated with high power electronics for clocking.
\end{abstract}

\section{INTRODUCTION}

As third and fourth generation $(3 \mathrm{G}, 4 \mathrm{G})$ wireless communications have emerged over the past few years the demand for higher bandwidth wireless data transfer has increased. This has placed great importance on the performance of radio base stations, requiring low phase-noise frequency sources that exhibit immunity to nearby interferers and excellent power handling [1]. With quality factors in the thousands at $\mathrm{GHz}$ frequencies, small footprints, and the ability to achieve multiple frequencies on the same chip, MEMS resonators can provide building blocks for low phase-noise oscillators operating over a wide frequency range.

Single-stage frequency sources are typically preferred for reduced cost, size, weight and power. As a wide bandgap semiconductor, GaN provides high electron velocities, charge densities $\left(1 \times 10^{13} \mathrm{~cm}^{-2}\right.$ in $\left.\mathrm{AlGaN} / \mathrm{GaN}\right)$, and critical electric fields, ideal for high power $(>10 \mathrm{~W} / \mathrm{mm})$, high frequency $(>300 \mathrm{GHz})$ integrated circuits. As a result, GaN High Electron Mobility Transistors (HEMTs) have been increasingly used for microwave monolithically integrated circuits (MMICs) and high power electronics. The ability to amplify and deliver higher power densities than gallium arsenide makes $\mathrm{GaN}$ a great candidate for use in single-stage oscillators without the need for additional amplifiers.

$\mathrm{GaN}$ also exhibits excellent electromechanical properties, such as high piezoelectric coefficients (electromechanical coupling $k_{T}^{2}$ up to $2 \%$ in FBARs [2]), high acoustic velocities and low acoustic losses. Hence, this technology offers a platform for seamless integration of high performance RF MEMS resonators with high power, high frequency electronics. Monolithic integration of MEMS resonators with ICs would lead to reduced matching constraints and parasitics, which ultimately translate into lower phasenoise and reduced jitter clocks, especially at high frequency.

For successful implementation in low phase-noise oscillators, these resonators must satisfy two requirements [1]: (1) High net signal-to-noise ratio and (2) small half-power bandwidth, i.e., high $Q$. For broadband operation, resonators must be scaled to multi$\mathrm{GHz}$ frequencies. At such high frequencies, passive resonators suffer from capacitive feed-through between drive and sense transducers which overwhelms the mechanical peak and lowers the signal-to-noise ratio (SNR). Active Field Effect Transistor (FET) sensing has been shown to overcome this challenge by providing internal electromechanical amplification of the resonant signal. In (a) Previous designs

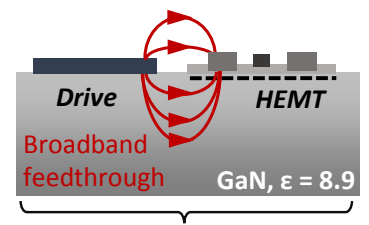

Single Resonant

Cavity (b) This work

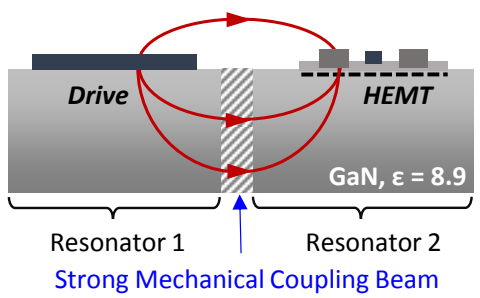

Figure 1: Schematic of GaN Resonant Body Transistor. (a) Placement of drive transducer and sense transistor in the same acoustic cavity leads to broadband feed-through. (b) Electrical isolation between drive transducer and sense transistor is done by mechanically coupling two resonators.

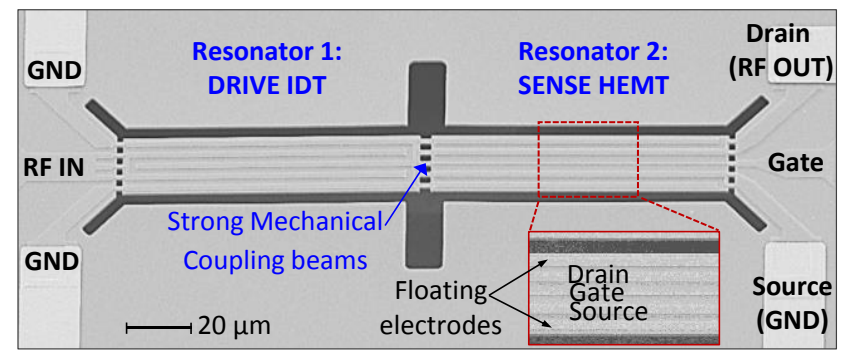

Figure 2: SEM of mechanically coupled drive and sense resonators. Source and drain electrodes, and two additional floating electrodes are patterned to match the drive transducer. A 5-tether approach is implemented to allow for a larger heat sink due to the HEMT current, as well as for improved power handling and spurious mode reduction [7]. Trenches are patterned through GaN to provide stress relief and better isolation between drive/sense.

Silicon, FET sensing has enabled resonators over $37 \mathrm{GHz}$ [3]. In $\mathrm{GaN}$, multi-GHz HEMT-sensed resonators have been demonstrated in contour mode [4] and thickness mode resonators [5]. These previously demonstrated RBTs implement a single resonant cavity containing both drive transducer and sense transistor, resulting in direct electrical coupling between the RF input and transistor terminals (Fig. 1(a)), which increases the broadband floor of the device. Instead, we propose an RBT in which drive transducer and sense transistor are embedded in two electrically isolated, mechanically coupled resonators (Fig. 1(b)). While mechanical coupling of two or more passive MEMS resonators has been previously used to achieve tunable, low insertion-loss filters [6], this is the first implementation that takes advantage of active transistor sensing.

\section{PRINCIPLE OF OPERATION}

Fig. 2 shows the SEM of a mechanically coupled GaN RBT. Acoustic waves are launched piezoelectrically with an interdigitated transducer (IDT) in resonator 1. This first resonator is mechanically coupled to resonator 2 , via an array of beams of length equal to half wavelength of the beam vibration at the desired resonant frequency. This guarantees that the two bars will resonate in phase [6]. A HEMT is embedded in resonator 2 for sensing. At resonance, standing waves are induced in both drive and sense resona- 
tors, leading to strain fields that modulate the carrier concentration of the 2D electron gas (2DEG) in the channel of the HEMT. Then these strains induce a polarization field through the direct piezoelectric effect:

$$
\begin{aligned}
& \overrightarrow{\boldsymbol{P}_{\boldsymbol{P E}}}=e_{51} \epsilon_{z x} \widehat{\boldsymbol{x}}+e_{51} \epsilon_{y z} \widehat{\boldsymbol{y}}+ \\
& +\left(e_{31} \epsilon_{z}+e_{31} \epsilon_{y}+e_{33} \epsilon_{z}\right) \hat{z},
\end{aligned}
$$

where $\epsilon_{x}, \epsilon_{y}$, and $\epsilon_{z}$ are the strains along the $\mathrm{x}, \mathrm{y}$ and $\mathrm{z}$-axis respectively and $\epsilon_{y z}$ and $\epsilon_{z x}$ are shear strains. Here, $e_{31}, e_{33}$ and $e_{51}$ are the piezoelectric coefficients corresponding to the Wurtzite crystal structure. This piezoelectric polarization induces straindependent bound charge throughout the $\mathrm{AlGaN} / \mathrm{GaN}$ heterostructure as discussed in $[4,8]$. As a result, free carriers flow from the Ohmic contacts to compensate for this piezoelectrically modulated charge which results in an AC modulation of the drain current.

The AC current modulation is represented in the equivalent small signal model in Fig. 3. In the electrical domain, this device can be decomposed into two branches. The RLC branch corresponds to the mechanical resonance induced in the two coupled bars, and is equivalent to a 1-port passive device. The second branch is a modified HEMT equivalent circuit, where the normal transistor transconductance is now replaced by an electromechanical transconductance, $g_{\alpha}$ [9]. The parasitic feed-through between the input and output of the RBT includes a capacitance, $C_{f t}$ and a substrate resistance, $r_{f t}$. This parasitic feed-through is what the mechanically coupled RBT design addresses, with an order of magnitude improvement from previously demonstrated RBTs that contain both drive and sense in the same resonant cavity.

While mechanically coupled RBTs can be implemented in various bulk or flexural modes, in this work we focus on the zero order symmetric Lamb mode, So. The authors have previously optimized and investigated the transduction capability of these modes in standard MMIC technology, achieving $Q s$ as high as 5500 and effective electromechanical coupling $\left(\mathrm{k}_{\mathrm{eff}}{ }^{2}\right)$ of $0.39 \%$ for resonators between 1-2 GHz [10]. Fig. 4(a) shows a 3D simulation of 2 mechanically coupled Lamb mode resonators. The 2D crosssection of the strain under the HEMT, with maximum modulation

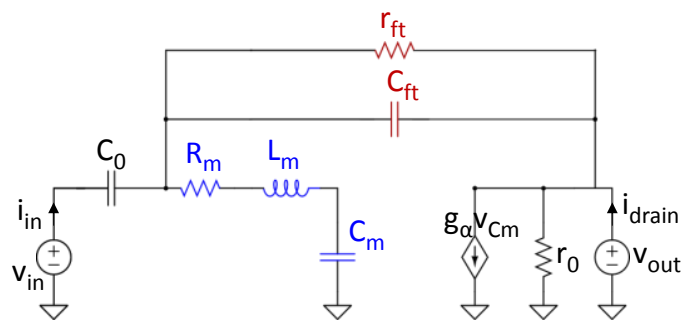

Figure 3: Equivalent circuit model of the mechanically coupled RBT. Blue elements represent the equivalent mechanical RLC branch, according to a Butterworth Van Dyke model while the right side captures the electromechanical transconductance.

(a)

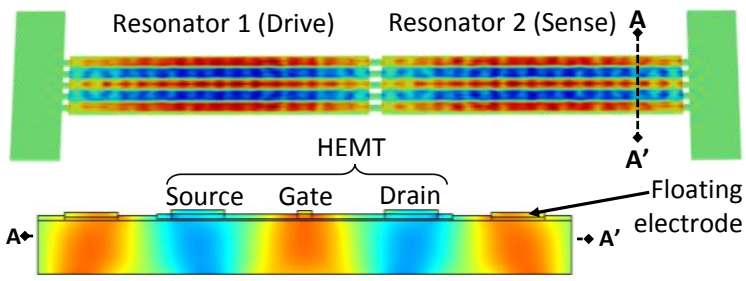

Figure 4: (a) 3D mode shape of strongly coupled resonators. (b) Gate of sense HEMT is placed at maximum strain. Source, drain and floating electrodes are patterned to match drive IDT. under the gate for optimal drain current modulation, is presented in Fig. 4(b). The gate of the embedded HEMT is designed at $500 \mathrm{~nm}$. Two floating electrodes are patterned in gate metal on either side of the HEMT in order to maintain mass loading symmetric to the drive IDT.

\section{FABRICATION}

Devices were fabricated at MIT using Raytheon's standard MMIC GaN-on-Si heterostructure, comprised of Molecular Beam Epitaxy (MBE) $\operatorname{AlGaN}(25 \mathrm{~nm}) / \mathrm{GaN}(1.7 \mu \mathrm{m})$ on (111)-Si using a thin AlN nucleation layer (Fig. 5(a)).

This is the first implementation of GaN MEMS resonators in a Au-free HEMT process flow. While typical HEMT MMIC processes use Au electrodes to make Ohmic contact to the 2DEG channel and for low-resistance transistor gates, many efforts have been made to realize Au-free metallization schemes in order to allow for the integration of GaN MMICs in CMOS foundries [11]. $\mathrm{Au}$-based Ohmic contacts involve a final anneal step at very high temperatures $\left(850-950^{\circ} \mathrm{C}\right)$ that allows the metal to diffuse and contact the 2DEG. As a result, the electrodes that undergo this step suffer from rough morphology and poor line acuity, which leads to yield and performance limitations in MEMS resonators, especially when scaling to higher frequencies. In the case of the RBT, where the HEMT is embedded in the resonant cavity, these rough Ohmic contacts can degrade mechanical performance. In this work we make use of Au-free Ohmic contacts that undergo a lower temperature anneal $\left(600^{\circ} \mathrm{C}\right)$. Eliminating Au-electrodes from the resonators also allows for higher resonator quality factors, since $\mathrm{Au}$ is known to cause additional dissipation through mass loading, phonon-electron scattering and interfacial losses.

We fabricate mechanically coupled RBTs in a standard GaN HEMT flow with only two additional steps. Processing starts with a shallow $\mathrm{AlGaN}$ etch in a $\mathrm{BCl}_{3} / \mathrm{Cl}_{2}$ chemistry which removes the $2 \mathrm{D}$ electron gas (2DEG) between the $\mathrm{AlGaN} / \mathrm{GaN}$ layers in areas where IDTs will be patterned. This allows for transduction through the entire volume of the GaN film. The 2DEG is only kept in the areas where HEMTs will be fabricated. Next, the Ohmic metal (5 $\mathrm{nm} \mathrm{Ta} / 100 \mathrm{~nm} \mathrm{Al} / 20 \mathrm{~nm} \mathrm{Ta}$ ) is deposited, and a 10 minute anneal at $600^{\circ} \mathrm{C}$ is used to drive the metal in to contact the 2DEG. The gate metal of the transistor $(100 \mathrm{~nm} \mathrm{Ni})$ is then patterned. This step is also used to define piezoelectric IDTs (Fig. 5(b)). A PECVD $\mathrm{Si}_{3} \mathrm{~N}_{4}$ layer $(180 \mathrm{~nm})$ is then deposited to passivate the surface and protect the 2DEG channel. One modification to the standard MMIC process involves a deep $\mathrm{Cl}_{2} \mathrm{GaN}$ etch in an inductively coupled plasma (Fig. 5(c)) which defines the acoustic cavities Metal pads $(50 \mathrm{~nm} \mathrm{Ti} / 300 \mathrm{~nm} \mathrm{Au})$ are then connected to the gate and Ohmic electrodes through vias in the passivation layer, for easy device probing. Finally, a $\mathrm{XeF}_{2}$ silicon etch releases the resonators (Fig. 5(d)).

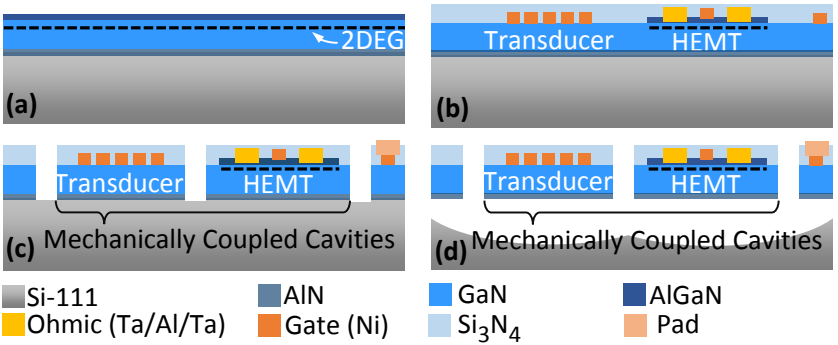

Figure 5: Mechanically coupled RBTs are fabricated in Au-free standard HEMT process with two additional steps for defining the acoustic cavity and for the final release. 


\section{EXPERIMENTAL RESULTS}

Mechanically coupled RBTs were measured in vacuum on a Cascade RF probe station using a standard 2-port measurement on an Agilent 5225A Network Analyzer, and de-embedded using an on-chip Open structure. For each of these devices, the electromechanical transconductance is defined as:

$$
g_{\text {em }}=Y_{21}-Y_{12}
$$

\section{Low-velocity coupling}

Fig. 6 plots the measured electromechanical transconductance, $g_{\text {em }}$, of the device shown in Fig. 2. The $1.012 \mathrm{GHz}$ resonance exhibits $Q$ of 3077 , with $f \cdot Q$ product of $3.1 \times 10^{12}$. The offresonance floor in this device is below $0.8 \mu \mathrm{S}$, which is $>10 \times$ lower than previously reported RBTs $[4,5]$. This corresponds to an SNR of 20.4. As discussed before, this broadband suppression is critical for the realization of low phase-noise oscillators, especially when scaling to $\mathrm{GHz}$ frequencies. The effect of reduced broadband floor can be seen in the inset of Fig. 6, which shows a clear $180^{\circ}$ shift at the mechanical resonance.

The DC behavior of the embedded HEMT used for electromechanical sensing is shown in Fig. 7(a). The transistor drives a maximum current of $12 \mathrm{~mA}$ in saturation, and has a threshold voltage of $-4 \mathrm{~V}$. The current degradation at drain bias $\left(\mathrm{V}_{\mathrm{DS}}\right)>2 \mathrm{~V}$ can be attributed to self-heating, as the HEMT is released from the substrate so the heat can only dissipate through the 5 anchoring tethers. In Fig. 7(b), the dependence of $g_{e m}$ on drain bias in the linear regime is shown, exhibiting a linear increase in electromechanical transconductance at resonance. It is important to note that the broadband floor remains constant independent of drain voltage. This behavior yields an increase in SNR from 7.6 for $\mathrm{V}_{\mathrm{DS}}=0.9 \mathrm{~V}$ to 13.6 for $\mathrm{V}_{\mathrm{DS}}=1.1 \mathrm{~V}$. In Fig. 7 (c), the effect of gate bias $\left(\mathrm{V}_{\mathrm{GS}}\right)$ on the electromechanical transconductance is shown for a fixed drain voltage. Optimal performance is obtained when $\mathrm{V}_{\mathrm{GS}}$ is set to $-2 \mathrm{~V}$.

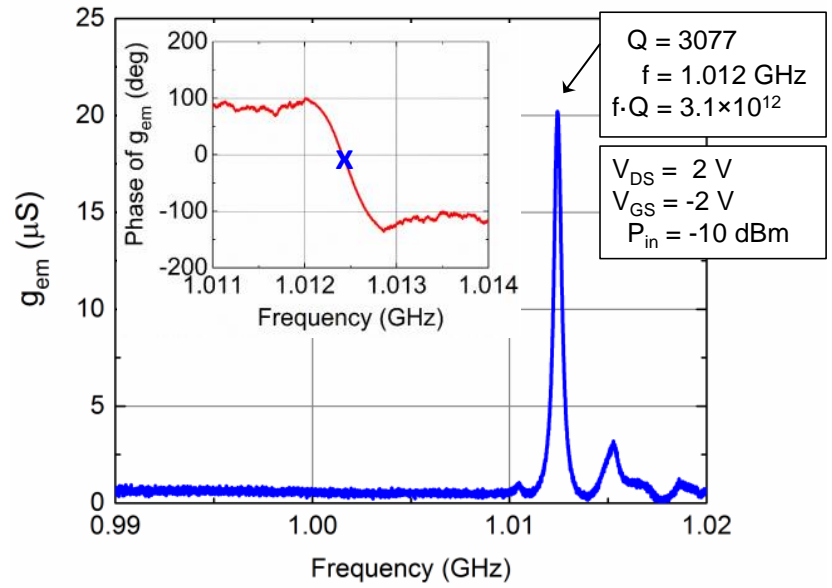

Figure 6: Measured $g_{\text {em }}=Y_{21}-Y_{12}$ of mechanically coupled RBT in Fig. 2 shows $>10 \times$ reduction in broadband floor compared to previously reported results. Inset shows $180^{\circ}$ phase shift corresponding to mechanical resonance.

\section{High-velocity coupled RBTS}

Fig. 8 presents an SEM of an RBT where the mechanical coupling between the drive and sense resonators is realized at the point of maximum velocity. This design opens up additional tethers on the sense resonator to enable routing for multi-finger HEMTs, necessary to achieve higher electromechanical transconductance, $g_{\mathrm{em}}$. To optimize performance, both gates of the multi-finger HEMT are placed at points of maximum strain. The 2-gate transistor reaches a saturation current of $30 \mathrm{~mA}$. The measured $g_{\text {em }}$ a)

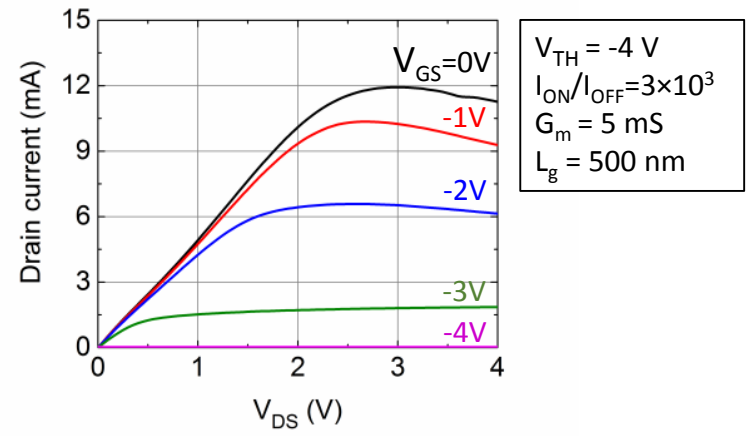

b)

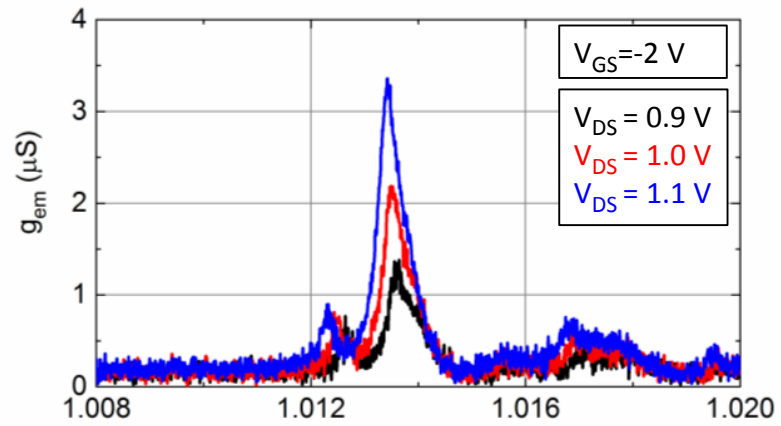

c)

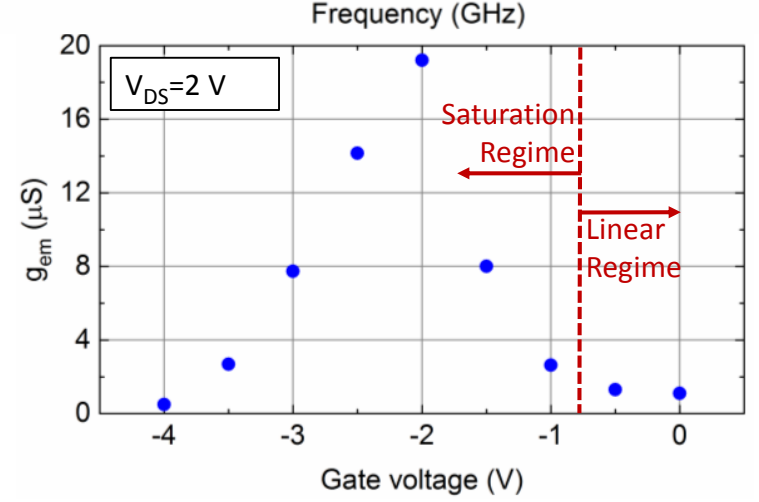

Figure 7: (a) DC behavior of embedded HEMT. (b) Dependence of $g_{\text {em }}$ on drain voltage in the linear regime shows linear increase in acoustic transconductance, while floor remains mainly unchanged. (c) Dependence of $g_{e m}$ on gate voltage in the saturation regime shows optimal performance when the gate is set to $-2 \mathrm{~V}$.

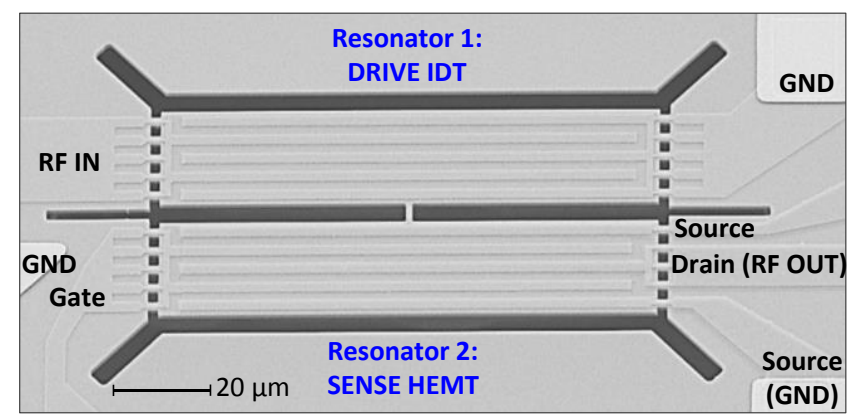

Figure 8: SEM of mechanically coupled RBT, with coupling beam at maximum velocity point. The embedded HEMT has two fingers, with gate length of $3.7 \mu \mathrm{m}$. Similar to the low-velocity case, trenches etched in GaN are used for stress relief and better isolation between drive/sense. An extra ground electrode is placed between the RF input and gate for better shielding. 


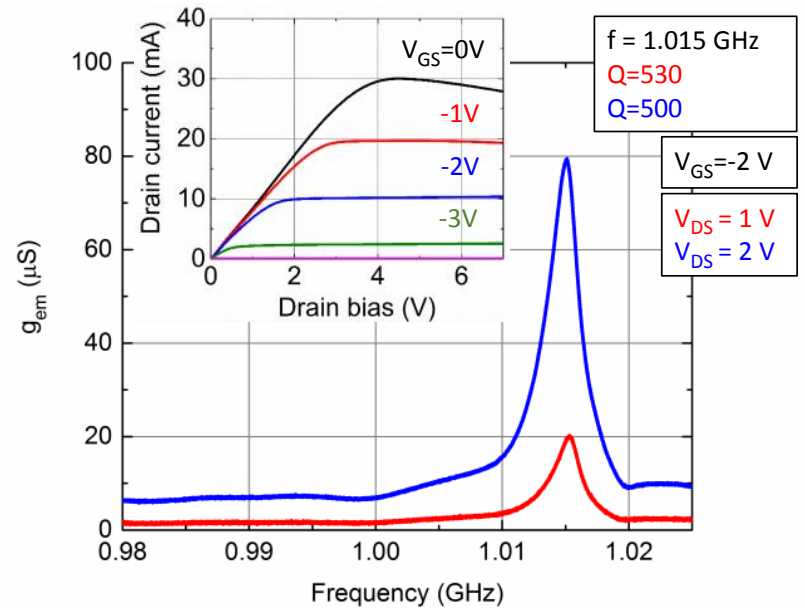

Figure 9: Measured $g_{\text {em }}$ for double-gate RBT shows an acoustic transconductance of $72 \mu S,>2 \times$ higher than previously reported GaN RBTs, with SNR > 50.

for two drain bias points is shown in Fig. 9. While there is a small change in the broadband floor with biasing conditions, the floor at $\mathrm{V}_{\mathrm{DS}}=2 \mathrm{~V}$ is still smaller than previously demonstrated devices, while the $g_{e m}$ of $72 \mathrm{uS}$ is the largest achieved in GaN RBTs to date. Possible reasons why this device provides less isolation than the low-velocity coupling case include spatial proximity of electrode routing outside the resonant structure, larger overlap area between the drive and sense cavity, and a higher overall transistor transconductance. However, the SNR for this device is higher than in the low-coupling case.

Table 1 summarizes the results for 3 devices described in this work, including 2 low-velocity coupled RBTs (one with $\mathrm{Lgate}_{\mathrm{g}}=3.7$ $\mu \mathrm{m}$ and the other shown in Fig. 1) and one high velocity coupled RBT (Fig 8). These resonators are compared with HEMT-sensed $\mathrm{GaN}$ resonators published in literature, and demonstrate order-ofmagnitude improvement in SNR.

Table 1: Summary of mechanical performance of state-of-the art GaN RBTs shows 100× improvement in SNR in this work.

\begin{tabular}{c|c|c|c|c|c|c|c|}
\cline { 2 - 8 } \multicolumn{1}{c|}{} & $\begin{array}{c}\mathrm{f} \\
(\mathrm{GHz})\end{array}$ & $\mathrm{Q}$ & $\begin{array}{c}\mathrm{G}_{\mathrm{M}} \\
(\mathrm{mS})\end{array}$ & $\begin{array}{c}\mathrm{I}_{\max } \\
(\mathrm{mA})\end{array}$ & $\begin{array}{c}\mathrm{g}_{\mathrm{em}} \\
(\mu \mathrm{S})\end{array}$ & $\begin{array}{c}\text { floor } \\
(\mu \mathrm{S})\end{array}$ & $\mathrm{SNR}$ \\
\hline \hline$[4]$ & 2.67 & 650 & 2.5 & 7.5 & 6 & 14 & 0.4 \\
\hline$[5]$ & 4.23 & 250 & 3 & 8 & 25 & 50 & 0.5 \\
\hline $\begin{array}{c}\text { Low } \\
\text { vel. 1 }\end{array}$ & 1.013 & 3068 & 4.3 & 11 & 9 & 0.8 & 11.3 \\
\hline $\begin{array}{c}\text { Low } \\
\text { vel. 2 }\end{array}$ & 1.013 & 3077 & 5 & 12 & 19 & 0.9 & 20.4 \\
\hline $\begin{array}{c}\text { High } \\
\text { vel. }\end{array}$ & 1.015 & 530 & 10 & 30 & 72 & 1.4 & 51.4 \\
\hline
\end{tabular}

\section{Frequency Tuning}

One consequence of HEMT sensing in the GaN RBT is selfheating of the suspended structure, which is thermally grounded only through small suspension beams. This self-heating leads to a change in the elastic constants of the resonant cavity, resulting in a frequency shift. A previous study by the authors found the temperature-coefficient of frequency of these $1 \mathrm{GHz}$ Lamb modes [10] to be $-24.2 \mathrm{ppm} /{ }^{\circ} \mathrm{C}$. Here, we investigate the frequency shift as a function of applied drain current, as shown in Fig. 10, with over $0.2 \%$ frequency shift at $6.6 \mathrm{~mA}$ bias current. The device provides a built-in microheater enabling tuning capabilities for frequency drift compensation due to temperature, packaging stresses, or other time-dependent parameters.

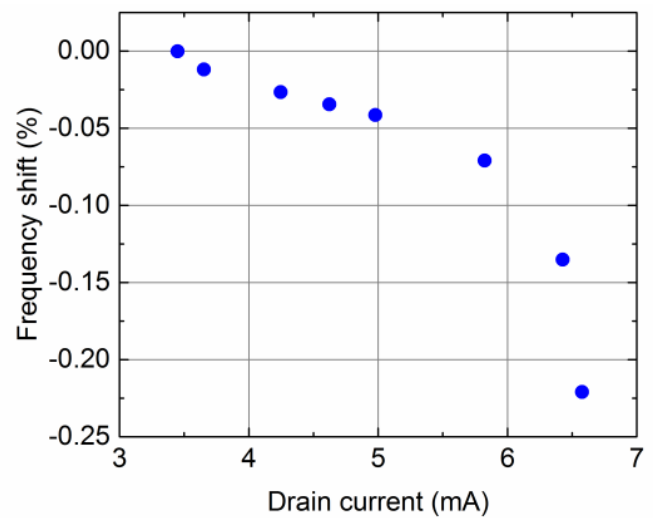

Figure 10: Measured resonant frequency shift with drain current for low-velocity coupling device shows $0.2 \%$ tuning capability.

\section{CONCLUSION}

We have demonstrated a method for suppressing offresonance feed-through in GaN RBTs, enabling >100x improvement in SNR. These devices were fabricated in a Au-free process which has yielded the highest $f \cdot Q$ products in GaN RBTs to date. At the same time, the RBTs presented here achieved $>2 \times$ higher acoustic transconductance than previous $\mathrm{GaN}$ devices. Realized in standard MMIC technology, these devices can leverage strong piezoelectric coupling, large breakdown fields, and high speed HEMTs for monolithically integrated, low phase-noise clocks.

\section{ACKNOWLEDGEMENTS}

We thank William Hoke, Brian Schultz and Thomas Kazior (Raytheon) for GaN growth. Fabrication took place at MIT's MTL under DARPA DAHI Foundry N66001-13-1-4022 and NSF Career ECCS-1150493 funding.

\section{REFERENCES}

[1] R. J. van de Plassche, J.H. Huijsing, W.M.C. Sansen, Analog Circuit Design, Kluwer Academic Publishers, Boston, 1997.

[2] A. Muller, D. Neculoiu, G. Konstantinidis, et. al., " $6.3 \mathrm{GHz}$ Film Bulk Acoustic Resonator structures based on Gallium Nitride/Silicon Thin Membrane", Elec. Dev. Lett., 30, 8 (2009).

[3] D. Weinstein, S.A. Bhave, "Acoustic Resonance in an independentgate FinFET,” Hilton Head 2010, pp.459-462.

[4] L.C. Popa, D. Weinstein, "Switchable Piezoelectric Transduction in AlGaN/GaN MEMS Resonators,” Transducers 2013, pp.2461-64.

[5] A. Ansari, M. Rais-Zadeh, "A Thickness-Mode AlGaN/GaN Resonant Body High Electron Mobility Transistor", Trans. Elec. Dev, 61, 4 (2014).

[6] F.D. Bannon, J.R. Clark, and C.T.-C. Nguyen, "High- $Q$ HF Microelectromechanical Filters", IEEE Journ. Sol. St. Circ, 35, 4 (2000).

[7] M. Shahmohammadi, B. Harrington, R. Abdolvand, "Concurrent Enhancement of Q and Power-handling in Multi-Tether High-Order Extensional Resonators", MTT, pp.1452-1455 (2010).

[8] M. Faucher, et al, "Amplified piezoelectric transduction of nanoscale motion in gallium nitride electromechanical resonators," Appl. Phys. Lett., 94, 233506 (2009).

[9] R. Marathe, B. Bahr, W. Wang et. al., "Resonant Body Transistors in IBM's 32 nm SOI Technology," J. MEMS, no. 99 (2013).

[10] L.C. Popa, and D. Weinstein, "L-band Lamb mode resonators in Gallium Nitride MMIC technology", to be presented at IFCS 2014.

[11] H.-S. Lee, D.S. Lee, T Palacios, "AlGaN/GaN High ElectronMobility Transistors Fabricated Through a Au-free Technology", Elec. Dev. Lett., vol. 32, no. 5, 2011.

\section{CONTACT}

*L.C. Popa, lpopa@mit.edu 